\title{
A Numerical Method for Reconstructing the Coefficient in a Wave Equation
}

\author{
Yat Tin Chow* $\quad$ Jun Zou ${ }^{\dagger}$
}

\begin{abstract}
We present a numerical method for reconstructing the coefficient in a wave equation from a single measurement of partial Dirichlet boundary data. The original inverse problem is converted to a nonlinear integral differential equation, which is solved by an iterative method. At each iteration, one linear second-order elliptic problem is solved to update the reconstruction of the coefficient, then the reconstructed coefficient is used to solve the forward problem to obtain the new data for the next iteration. The initial guess of the iterative method is provided by an approximate model. This model extends the approximate globally convergent method proposed by Beilina and Klibanov, that has been well developed for the determination of the coefficient in a special wave equation. Numerical experiments are presented to demonstrate the stability and robustness of the proposed method with noisy data.
\end{abstract}

Key Words. Wave equation, reconstruction, single measurement.

MSC classifications. 35R30, 74G75, 65R32.

\section{Introduction}

We shall develop a numerical method for an inverse problem arising from reconstructing the coefficient in the following wave equation

$$
\frac{\partial^{2} u}{\partial t^{2}}=\nabla \cdot(\mu \nabla u) \quad \text { in } \quad \mathbb{R}^{d} \times(0, \infty)
$$

where $u$ may be the displacement or pressure of some physical medium and the coefficient $\mu$ may represent the physical properties of the medium. This inverse problem is involved when one tries to identify the locations and physical properties of inhomogeneous media sitting inside a homogeneous background medium. Such a technique is very useful and may have several physical applications. The wave model (1.1) may be considered as a special case of the time-dependent transverse magnetic polarized wave scattering problem [13], or as a simplified acoustic wave model for fluids with variable density and constant bulk modulus [5, 14, 15]. It may also be viewed as a simplified model for the shear waves in a two-dimensional isotropic elastic medium when the scalar displacement travels along the direction transversal to the medium. In recent

\footnotetext{
*Department of Mathematics, The Chinese University of Hong Kong, Shatin, Hong Kong. (ytchow@math.cuhk.edu.hk).

${ }^{\dagger}$ Department of Mathematics, The Chinese University of Hong Kong, Shatin, N.T., Hong Kong. (zou@math.cuhk.edu.hk).
} 
years, some rapid identification techniques have been developed for solving the elastodynamic inverse problem, for instance, crack/fault identification techniques are developed for cracks having free boundary condition using a reciprocity gap function [6, 7], and linear sampling techniques are designed to locate inclusions in the isotropic elastic medium $[4,12]$. In this work we shall mainly focus on the inverse problem of reconstructing the coefficient $\mu$ in the model equation (1.1), using some Dirichlet boundary data from a single measurement. We will develop a numerical method based on the approximate globally convergent method proposed by Beilina and Klibanov in [1], which will be referred to as the Beilina-Klibanov method.

The least-squares minimisation combined with a Tikhonov regularization is a popular technique to solve a multi-dimensional coefficient inverse problem (MCIP). However, this technique may encounter a common barrier to numerical solutions of inverse problems, i.e., the iterative optimization process may be trapped in the neighborhoods of some local minima. This difficulty may be overcome when a good initial guess is available, but it may not be always convenient and possible to achieve a reasonable initial guess. An intriguing and very challenging goal is to construct a numerical method which would deliver a good approximation to the exact solution without any a priori knowledge of a small neighborhood of this solution. The development of the Beilina-Klibanov method aims to reach this goal by proposing a method which does not rely on the construction of a least-squares functional, instead it makes full use of the structure of the underlying forward model.

The Beilina-Klibanov method for MCIPs was first proposed in [1], and further developed in many follow-up studies. The detailed developments of this method and its convergence analysis are available in a recent monograph [3]. The main advantage of this method is that, within the framework of a reasonable approximation, it delivers a good approximation to the true solution without any advanced knowledge of a small neighborhood of this solution. Indeed, it is well known that MCIPs are highly nonlinear and ill-posed. In the case of a single measurement, as considered by the Beilina-Klibanov method and also in our current work, the amount of data is minimized.

The Beilina-Klibanov method has been well developed so far only for the hyperbolic equation

$$
c(x) \frac{\partial^{2} u}{\partial t^{2}}=\Delta u
$$

with the unknown coefficient $c(x)>0$, where $c(x)$ is sitting outside all derivatives. The governing model equation (1.1) investigated in this work is quite different from (1.2) and more challenging for its numerical inversions. This work explores the possibility to extend the BeilinaKlibanov method to the wave equation (1.1).

The rest of the paper is arranged as follows. The forward and inverse problems of our interest are introduced in section 2. A nonlinear integral differential equation is derived in section 3, which is crucial to the first step of the new numerical reconstruction algorithm. In section 4 we show how to obtain the first approximation to the so-called "tail function" and formulate our new algorithm in section 5. Some numerical experiments are presented in section 6.

\section{The forward and inverse problems}

In this work, we shall be concerned with the inverse problem of reconstructing the coefficient $\mu$ in the wave equation (1.1). Consider an inhomogeneous medium $\Omega$, an open bounded domain 
sitting in the homogeneous background $\mathbb{R}^{d}$. We assume that the coefficient $\mu(x)$ is piecewise $C^{1}$-smooth such that

$$
0<\beta \leq \mu(x) \leq \mu_{0} \quad \text { in } \quad \mathbb{R}^{d} ; \quad \mu(x)=\mu_{0} \quad \text { in } \quad \mathbb{R}^{d} \backslash \Omega,
$$

or

$$
\mu_{0} \leq \mu(x) \leq \gamma<\infty \quad \text { in } \quad \mathbb{R}^{d} ; \quad \mu(x)=\mu_{0} \quad \text { in } \quad \mathbb{R}^{d} \backslash \Omega .
$$

For sake of exposition, we assume that the incident plane wave propagates along some axis. For definiteness, this propagating axis is specified to be $x_{2}$-axis. Then we may complement equation (1.1) with the following initial conditions:

$$
u(x, 0)=0 \quad \text { and } \quad \frac{\partial u}{\partial t}(x, 0)=\delta\left(x_{2}-x_{02}\right)
$$

where $x_{02} \in \mathbb{R}$ is a fixed point. Furthermore, it is reasonable for us to assume that the plane wave is excited outside the inhomogeneous medium region $\Omega$, namely $\bar{\Omega} \cap\left\{x_{2}=x_{02}\right\}=\varnothing$.

Alternatively, we may also consider the case where a plane wave is generated along a line/plane $\left\{x_{2}=x_{02}\right\}$, namely

$$
\frac{\partial^{2} u}{\partial t^{2}}=\nabla \cdot(\mu \nabla u)+\delta\left(x_{2}-x_{02}\right) r(t) \quad \text { in } \quad \mathbb{R}^{d} \times(0, \infty),
$$

where $r(t)$ is the source strength at time $t$. Correspondingly we can complement equation (1.1) with the following initial conditions:

$$
u(x, 0)=0 \quad \text { and } \quad \frac{\partial u}{\partial t}(x, 0)=0 .
$$

In the case of a uniform plane wave, it is known that the phase speed of the wave is $\sqrt{\mu}$. Condition (2.1) states a case when the inclusions concerned is with a lower wave speeds than that of the background. On the other hand, condition (2.2) states a case when one detects some inclusions having a faster wave speed than the background.

\subsection{The forward problem in Laplace domain}

The Beilina-Klibanov method is constructed based on the governing forward equation in the pseudo-frequency domain. For this aim, we consider the Laplace transform of function $u(x, t)$ :

$$
w(x, s)=\int_{0}^{\infty} u(x, t) e^{-s t} d t \quad \forall s>\underline{s}
$$

where $\underline{s}$ is a positive constant such that the above integral converges. The parameter $s$ is called the pseudo-frequency. By applying the Laplace transform to the system (1.1) and (2.3) we obtain the wave equation in the pseudo-frequency domain:

$$
\nabla \cdot(\mu \nabla w)-s^{2} w=-\delta\left(x_{2}-x_{02}\right) \quad \forall x \in \mathbb{R}^{d}, \quad s \geq \underline{s} .
$$

It was proved in [3] (Theorem 2.7.1) that $\lim _{|x| \rightarrow \infty} w(x, s)=0$ if $s$ is sufficiently large, when $\delta\left(x_{2}-\right.$ $\left.x_{02}\right)$ is replaced by a point source of the form $\delta\left(x-x_{0}\right)$. In this work we shall assume that

$$
\lim _{|x| \rightarrow \infty}\left[w(x, s)-w_{0}(x, s)\right]=0 \quad \forall s>\underline{s}
$$


for sufficiently large $\underline{s}$, where $w_{0}$ is the fundamental solution to the equation $\Delta w_{0}-s^{2} w_{0}=$ $-\delta\left(x_{2}-x_{02}\right)$, namely

$$
w_{0}(x, s)=\frac{\exp \left(-s\left|x_{2}-x_{02}\right|\right)}{2 s} .
$$

\subsection{The inverse problem}

We are now going to formulate the inverse problem of our interest in both the time domain and the pseudo-frequency domain. Let $\Gamma$ be a non-empty relatively open subset of $\partial \Omega$, where we shall assume that the solution $u(x, t)$ is measured.

Inverse problem in time domain. Given the following measurement data:

$$
u=g(x, t) \quad \text { on } \quad \Gamma \times(0, T),
$$

determine the coefficient $\mu$ in the following wave system:

$$
\begin{aligned}
\frac{\partial^{2} u}{\partial t^{2}} & =\nabla \cdot(\mu \nabla u) \quad \text { in } \quad \mathbb{R}^{d} \times(0, \infty), \\
u(x, 0) & =0, \quad \frac{\partial u}{\partial t}(x, 0)=\delta\left(x_{2}-x_{02}\right) .
\end{aligned}
$$

Inverse problem in pseudo-frequency domain. Given the following boundary data:

$$
w(x, s)=\int_{0}^{\infty} g(x, \tau) e^{-s \tau} d \tau=: \phi(x, s) \text { on } \Gamma,
$$

determine the coefficient $\mu(x)$ in the following wave system in the pseudo-frequency domain:

$$
\begin{gathered}
\nabla \cdot(\mu \nabla w)-s^{2} w=-\delta\left(x_{2}-x_{02}\right) \quad \text { in } \quad \mathbb{R}^{d} \times(\underline{s}, \infty), \\
\lim _{|x| \rightarrow \infty}\left[w(x, s)-w_{0}(x, s)\right]=0 \quad \forall s>\underline{s} .
\end{gathered}
$$

The fact that the time interval is infinite in (2.12) is not a serious restriction. In fact, noting that the kernel of the Laplace transform integral decays rapidly with $\tau \rightarrow \infty$, only a small portion of the interval $(0, \infty)$ provides an essential contribution to this integral. Hence, only the data of $g(x, t)$ on this small portion are important or required in numerical reconstructions.

\section{A nonlinear integral differential equation}

In this section we develop a numerical reconstruction method for the inverse problem we formulated in section 2.2. To do so, we extend the basic idea of the Beilina-Klibanov method developed for equation (1.2) to the wave equation (1.1) and derive a crucial integral differential equation. Noting the fact that the line/plane $\left\{x_{2}=x_{02}\right\}$ is outside the inhomogeneous medium region $\Omega$, we obtain from (2.7) the equation

$$
\mu^{-1}\left(s^{2} w\right)=\Delta w+\nabla \ln \mu \cdot \nabla w \quad \text { in } \quad \Omega .
$$

If we define a new function $v:=(\ln w) / s^{2}$, then $v$ satisfies

$$
\Delta v+s^{2}|\nabla v|^{2}+\nabla \ln \mu \cdot \nabla v=\frac{1}{\mu} .
$$


Clearly this equation may help us find the unknown $\mu$ when $v$ is known. However, $v$ is not known a priori. Therefore we shall work out some technique to solve $v$ first. This leads to a fundamental integral differential equation which we intend to derive below.

The following asymptotic relation was established for the problem (3.1) under some appropriate conditions $[1,3]$ :

$$
\left\|\partial_{s}^{k} v(x, s)-(-1)^{k} \frac{p(x)}{s^{k+1}}\right\|_{C^{2+\alpha}(\bar{\Omega})}=O\left(\frac{1}{s^{k+2}}\right) \quad \text { as } \quad s \rightarrow \infty
$$

for $k=0,1$ and some smooth function $p$ in $\bar{\Omega}$. Now we introduce a function $q=\partial_{s} v$. Using the decay property of $q=\partial_{s} v$ in (3.2), we can write

$$
v(x, s)=-\int_{s}^{\bar{s}} q(x, \tau) d \tau-\int_{\bar{s}}^{\infty} q(x, \tau) d \tau:=-\int_{s}^{\bar{s}} q(x, \tau) d \tau+V(x, \bar{s})
$$

for $\bar{s}>>1$, and $V(x, \bar{s})$ satisfies

$$
V(x, \bar{s})=\frac{\ln w(x, \bar{s})}{\bar{s}^{2}}=v(x, \bar{s}) .
$$

The parameter $\bar{s}$ above is called the truncated pseudo-frequency and will be viewed as the regularization parameter in our new method. The fact that $\bar{s}$ can be treated as a regularization parameter will be explained more in Section 6 . The function $V(x, \bar{s})$ is called the tail function. Differentiating both sides of (3.1) with respect to $s$ and using (3.3)-(3.4), we obtain

$$
\begin{gathered}
\Delta q-2 s^{2} \nabla q \cdot \int_{s}^{\bar{s}} \nabla q(x, \tau) d \tau+2 s^{2} \nabla q \cdot \nabla V+2 s\left|\nabla \int_{s}^{\bar{s}} q(x, \tau) d \tau\right|^{2} \\
-4 s \nabla V \cdot \int_{s}^{\bar{s}} \nabla q(x, \tau) d \tau+2 s|\nabla V|^{2}+\nabla \ln \mu \cdot \nabla q=0,
\end{gathered}
$$

where $q$ admits the boundary condition

$$
q(x, s)=\psi(x, s):=\frac{\partial}{\partial s}\left(\frac{\ln \phi(x, s)}{s^{2}}\right) \quad \text { on } \quad \partial \Omega .
$$

Both $q$ and $V$ need to be solved in the equation (3.5). To do so, we follow $[1,3]$ to use the layer stripping technique and approximate $q$ and $V$ iteratively in the pseudo-frequency direction. First we partition the pseudo-frequency range $[\underline{s}, \bar{s}]$ by

$$
\underline{s}=s_{N}<s_{N-1}<\ldots<s_{3}<s_{2}<s_{1}<s_{0}=\bar{s}
$$

with a uniform stepsize $h$, then approximate $q$ by a piecewise constant function with respect to this partition, namely $q(x, s)=q_{n}(x)$ for $s \in\left(s_{n}, s_{n-1}\right]$ for $n=N, N-1, \cdots, 1$. By setting $q_{0}=0$, we can naturally have the following approximation

$$
\int_{s}^{\bar{s}} \nabla q(x, \tau) d \tau \approx\left(s_{n-1}-s\right) \nabla q_{n}(x)+h \sum_{j=0}^{n-1} \nabla q_{j}
$$


for $s \in\left(s_{n}, s_{n-1}\right)$. Now in (3.5), we replace $q$ by its piecewise constant approximation and the integral term by the approximation (3.7), then multiply both sides of the equation by a Carleman weight function $e^{\lambda\left(s-s_{n-1}\right)}$ for some $\lambda>>1$ (to be selected), and then integrate the resulting equation with respect to $s$ over $\left(s_{n}, s_{n-1}\right)$ to obtain

$$
\begin{aligned}
& \Delta q_{n}+A_{3, n}\left|\nabla q_{n}\right|^{2}-A_{1, n} \nabla q_{n} \cdot h \sum_{j=0}^{n-1} \nabla q_{j}+A_{1, n} \nabla q_{n} \cdot \nabla V_{n} \\
& +2 h^{2} A_{2, n}\left|\sum_{j=0}^{n-1} \nabla q_{j}\right|^{2}-4 A_{2, n} \nabla V_{n} \cdot h \sum_{j=0}^{n-1} \nabla q_{j}+2 A_{2, n}\left|\nabla V_{n}\right|^{2}+\nabla \ln \mu \cdot \nabla q_{n}=0,
\end{aligned}
$$

where the coefficients $A_{1, n}, A_{2, n}$ and $A_{3, n}$ have the following closed forms:

$$
\begin{aligned}
& A_{1, n}=\left(\int_{s_{n}}^{s_{n-1}} e^{\lambda\left(s-s_{n-1}\right)} d s\right)^{-1}\left(\int_{s_{n}}^{s_{n-1}}\left(2 s^{2}-4 s\left(s_{n-1}-s\right)\right) e^{\lambda\left(s-s_{n-1}\right)} d s\right), \\
& A_{2, n}=\left(\int_{s_{n}}^{s_{n-1}} e^{\lambda\left(s-s_{n-1}\right)} d s\right)^{-1}\left(\int_{s_{n}}^{s_{n-1}} s e^{\lambda\left(s-s_{n-1}\right)} d s\right), \\
& A_{3, n}=\left(\int_{s_{n}}^{s_{n-1}} e^{\lambda\left(s-s_{n-1}\right)} d s\right)^{-1}\left(\int_{s_{n}}^{s_{n-1}}\left(-2 s^{2}\left(s_{n-1}-s\right)+2 s\left(s_{n-1}-s\right)^{2}\right) e^{\lambda\left(s-s_{n-1}\right)} d s\right) .
\end{aligned}
$$

Using (3.6), we may impose the following Dirichlet condition for the solution $q_{n}$ in (3.8):

$$
q_{n}(x)=\psi_{n}(x):=\frac{1}{h}\left(\frac{\ln w\left(x, s_{n-1}\right)}{s_{n-1}^{2}}-\frac{\ln w\left(x, s_{n}\right)}{s_{n}^{2}}\right) \quad \text { on } \quad \partial \Omega .
$$

As the coefficient $A_{3, n}$ decays as $\lambda \rightarrow \infty[1,3]$, we shall drop the second term in (3.8) from now on. We remark that the tail function $V$ in (3.5) is replaced by an approximation $V_{n}$ in (3.8).

If the data is available only on the partial boundary $\Gamma$ on $\partial \Omega$, we may assume $w$ takes the value of the fundamental solution $w_{0}$ on the rest of the boundary, i.e., $\partial \Omega \backslash \Gamma$. Thus the boundary condition (3.9) changes to

$$
\left.q_{n}\right|_{\Gamma}=\psi_{n}(x) \quad \text { and }\left.\quad q_{n}\right|_{\partial \Omega \backslash \Gamma}=\frac{1}{h}\left(\frac{\ln w_{0}\left(x, s_{n-1}\right)}{s_{n-1}^{2}}-\frac{\ln w_{0}\left(x, s_{n}\right)}{s_{n}^{2}}\right) .
$$

This was done in a similar manner in [3] (section 6.8.5), and it is reasonable if the inclusions in $\Omega$ do not essentially affect the propagation of the wave on the partial boundary $\partial \Omega \backslash \Gamma$. This is the case of our interest in this work, where the backscattering data is collected along the partial boundary $\Gamma$. The choice of the above boundary data will be justified numerically in Example 2 of section 6 .

\section{Approximation of the first tail}

Now we discuss how to find the unknown functions $q_{n}$ and the approximate tail functions $V_{n}$ in (3.8). A natural idea is to update $q_{n}$ and $V_{n}$ alternatively. We shall update function $q_{n}$ in an inner iteration inside the domain $\Omega$ from equation (3.8), while update function $V_{n}$ in an outer iteration from the system $(2.7)-(2.8)$ in the entire space $\mathbb{R}^{d}$ for each new iterate $\mu_{n}(x)$ of the 
unknown coefficient $\mu(x)$. Using equation (3.1), the coefficient $\mu_{\text {new }}$ can be improved from $\mu_{\text {old }}$ whenever $q_{n}, V_{n}$ and $v_{n}$ are available:

$$
\frac{1}{\mu_{\text {new }}(x)}=\Delta v_{n}\left(x, s_{n}\right)+s_{n}^{2}\left|\nabla v_{n}\left(x, s_{n}\right)\right|^{2}+\nabla\left(\ln \mu_{\text {old }}(x)\right) \cdot \nabla v_{n}\left(x, s_{n}\right) .
$$

In order to update $q_{n}$ and $V_{n}$ in (3.8), we need to find an appropriate initial approximation $V_{1,1}(x, \bar{s})$ of the first tail $V_{1}(x, \bar{s})$. To do this, we set $s=\bar{s}$ in equation (3.5) to obtain

$$
\Delta q+2 \bar{s}^{2} \nabla q \cdot \nabla V+2 \bar{s}|\nabla V|^{2}+\nabla \ln \mu \cdot \nabla q=0 \quad \text { in } \quad \Omega .
$$

From (3.4), we see that $V(x, \bar{s})=\frac{\ln w(x, \bar{s})}{\bar{s}^{2}}=v(x, \bar{s})$. Clearly, we can also view the tail $V(x, \bar{s})$ as a function of $x$ and $\bar{s}$, and sometimes we may simply write $V(x, s)$ when it is convenient. It then follows from the asymptotic behaviors (3.2) that the tail function $V(x, s)$ satisfies

$$
\partial_{s}^{k} V(x, s)=(-1)^{k} \frac{p(x)}{s^{k+1}}+O\left(\frac{1}{s^{k+2}}\right) \quad \text { as } \quad s \rightarrow \infty
$$

for $k=0,1$. So it is natural for us to propose the following initial approximation for the first tail function $V_{1}$ :

$$
V_{1,1}(x, s):=\frac{p(x)}{s} \quad \forall s \geq \bar{s}
$$

where $p$ is a function of $x$ to be determined. Under this approximate model, we can also suggest an initial approximation of function $q$. Noting that the original functions $q$ satisfies $q=\partial_{s} V$, we may naturally define the approximation of function $q$ by

$$
\tilde{q}(x, s):=\partial_{s} V_{1,1}(x, s)=\partial_{s}\left(\frac{p(x)}{s}\right)=-\frac{p(x)}{s^{2}} \quad \forall s \geq \bar{s}
$$

By substituting (4.4) and (4.5) into (4.2) we obtain

$$
0=\Delta\left(-\frac{p(x)}{\bar{s}^{2}}\right)+2 \bar{s}^{2} \nabla\left(-\frac{p(x)}{\bar{s}^{2}}\right) \cdot \nabla\left(\frac{p(x)}{\bar{s}}\right)+2 \bar{s}\left|\nabla\left(\frac{p(x)}{\bar{s}}\right)\right|^{2}+\nabla \ln \mu \cdot \nabla\left(-\frac{p(x)}{\bar{s}^{2}}\right),
$$

which gives

$$
\Delta p=-\nabla \ln \mu \cdot \nabla p .
$$

Noting the relations (3.6) and (4.5) we may impose the following boundary condition for $p$ :

$$
p=-\bar{s}^{2} \psi(x, \bar{s}) \quad \text { on } \quad \partial \Omega .
$$

Now it follows from (4.4) that the first initial tail $V_{1,1}(x, s)$ can be obtained by solving

$$
\Delta V_{1,1}(x)+\nabla \ln \mu \cdot \nabla V_{1,1}(x)=0 .
$$

If we assume only partial boundary data on $\Gamma \subset \partial \Omega$, we may take the following boundary condition for $V_{1,1}(x)$ :

$$
V_{1,1}(x)=\frac{\ln w\left(x, \bar{s}^{2}\right)}{\bar{s}^{2}} \text { on } \Gamma \quad \text { and } \quad V_{1,1}(x)=\frac{\ln w_{0}(x, \bar{s})}{\bar{s}^{2}} \text { on } \partial \Omega \backslash \Gamma,
$$

where $w_{0}$ is the fundamental solution. From the above we see that we can obtain an initial approximate tail $V_{1,1}$ from the system (4.8)-(4.9) for some initial guess of $\mu$. 


\section{A numerical reconstruction algorithm}

In this section we propose a new numerical reconstruction algorithm for the reconstruction of the coefficient $\mu(x)$ in (1.1) based on the derivations in sections 3.2-3.3, more accurately on the equations (3.1), (3.8)-(3.9) and (4.8)-(4.9). We see from (3.1) that function $\mu(x)$ can be reconstructed inside the sampling domain $\Omega$. But we need to solve the problem (2.7)-(2.8) in the entire space $\mathbb{R}^{d}$ to update the tail function. For the purpose we will extend each approximation of $\mu$ from $\Omega$ onto the entire space $\mathbb{R}^{d}$. As the background medium is homogeneous with a constant coefficient $\mu_{0}$, we will do the extensions naturally by the constant function $\mu_{0}$.

Now we are ready to formulate our reconstruction algorithm. The reconstruction is proceeded in a larger computational domain $G$ such that $\Omega \subset G$.

\section{Reconstruction Algorithm}

Step 0 . Choose an initial guess $\mu_{0}$, two stopping tolerances $\varepsilon_{1}, \varepsilon_{2}$, stepsize $h$, and set $\mu_{1,0}:=\mu_{0}$; Step 1. Solve the system (4.8)-(4.9) for the initial tail function $V_{1,1}$. Set $q_{0}:=0, n:=1, k:=1$. Step 2. Solve the system (3.8) and (3.10) for $q_{n, k}$ with $\mu:=\mu_{n, k-1}$ and $V_{n}:=V_{n, k}$;

Update $v_{n, k}$ (based on (3.3) and its approximation similar to (3.7)):

$$
v_{n, k}=-h q_{n, k}-h \sum_{j=0}^{n-1} q_{j}+V_{n, k} .
$$

Compute $\mu_{n, k}$ from the equation (4.1), and extend it from $\Omega$ onto $G$.

Step 3. Solve the problem (2.7) for $w_{n, k+1}$ with coefficient $\mu:=\mu_{n, k}$;

Update the tail function $V_{n, k+1}(x)=\left(\ln w_{n, k+1}(x, \bar{s})\right) / \bar{s}^{2}$.

If $\left\|V_{n, k+1}-V_{n, k}\right\|>\varepsilon_{1}$, set $k:=k+1$ and GOTO Step 2;

Otherwise set $V_{n+1,1}:=V_{n, k+1}, \mu_{n}:=\mu_{n, k}$ and $\mu_{n+1,0}:=\mu_{n}, q_{n}:=q_{n, k}$;

If $\left\|\mu_{n}-\mu_{n-1}\right\| \leq \varepsilon_{2}$, STOP; otherwise set $n:=n+1, k:=1$, GOTO Step 2 .

Remark 1 In our algorithm, (4.1) is used for the reconstruction of images $\mu_{n, k}$. This step may cause a possible loss of regularity of the image at each iteration. In order to minimize any possible impact of successive loss of regularity, we smooth the image $\mu_{n, k}$ by convolving it with a standard mollifier $K$ vanishing outside the unit sphere $B_{1}(0)$ (with a given $\epsilon>0$ )

$$
\tilde{\mu}_{n, k}(x):=\frac{1}{\epsilon^{d}} \int_{\mathbb{R}^{d}} K\left(\frac{x-y}{\epsilon}\right) \mu_{n, k}(y) d y .
$$

\section{$6 \quad$ Numerical experiments}

In this section we present a few numerical examples to test the performance of the newly proposed Reconstruction Algorithm in section 5. We choose the computational domain and the sampling domain to be $G=[0,1]^{2}$ and $\Omega=[0.25,0.75]^{2}$ respectively, and the exact coefficient $\mu$ to be 1 in the homogeneous background of $G$, and inhomogeneous in the sampling domain $\Omega$. We then place a few inhomogeneous inclusions inside the sampling domain $\Omega$ and apply the new algorithm to recover the inhomogeneities in $\Omega$.

Before we start the inversion process, we need to first solve the forward problem (1.1) on the entire space $\mathbb{R}^{d}$ to generate the observation data. To do so, we should truncate the infinite 
domain $\mathbb{R}^{d}$, approximated by a finite computational domain $G$, and introduce some boundary conditions on the artificial boundaries of $G$. We separate the boundary $\partial G$ in two parts, $\partial_{1} G=$ $\left(\left\{x_{2}=1\right\} \cup\left\{x_{2}=0\right\}\right) \cap \bar{G}$ and $\partial_{2} G=\left(\left\{x_{1}=1\right\} \cup\left\{x_{1}=0\right\}\right) \cap \bar{G}$, and apply the first-order absorbing boundary conditions on $\partial_{1} G$ [11]. We choose the incident plane wave that propagates in $x_{2}$-direction, and use the homogenous Neumann boundary condition on $\partial_{2} G$. This leads us to solve the following system for the expected measurement data:

$$
\begin{aligned}
\frac{\partial^{2} u}{\partial t^{2}} & =\nabla \cdot(\mu \nabla u)+\delta\left(x_{2}-x_{02}\right) r(t) \text { in } G \times(0, T), \\
u(x, 0) & =\frac{\partial u}{\partial t}(x, 0)=0 \text { in } G, \\
\frac{\partial u}{\partial \nu}-\frac{\partial u}{\partial t} & =0 \text { on } \partial_{1} G, \\
\frac{\partial u}{\partial \nu} & =0 \text { on } \partial_{2} G
\end{aligned}
$$

where we set $T=2, x_{02} \in(0.75,1)$, and $r(t)$ to be (with $\left.\omega=1 / 5\right)$

$$
r(t)= \begin{cases}\sin \left(\frac{2 \pi}{\omega} t\right) & \text { if } t \in(0, \omega), \\ 0 & \text { if } t>\omega .\end{cases}
$$

The forward problem (6.1) is solved in the computational domain $G$ with the given coefficient $\mu$ by using the explicit second order central difference scheme in time but the piecewise linear finite element method in space. The finite element method is used here for the space discretization of the system (6.1) as it is more convenient to handle possible jumps of the given coefficient $\mu$ in the system [8]. System (6.1) is solved in $G$ with a very fine mesh of $\Delta x=1 / 256$ and a very small time step $\Delta t=5 \times 10^{-4}$. Then the values of the solution to the forward problem on $\Gamma=\left\{\left(x_{1}, x_{2}\right) ; x_{2}=0.25,0.25 \leq x_{1} \leq 0.75\right\}$ are taken as the measurement data. 16 measurement points are taken along the boundary $\Gamma$ for the collection of measurement data. To test the robustness of the reconstruction algorithm against noise, we introduce some multiplicative noise to the data along $\Gamma$ in the time domain:

$$
u^{\delta}(x, t)=u(x, t)+\varepsilon \zeta(x, t) u(x, t) \quad \forall x \in \Gamma, t \in(0, T)
$$

where $\varepsilon$ is the noise level, and $\zeta(t)$ is a random variable uniformly distributed in $[-1,1]$. We shall take $\varepsilon=5 \%$ in our numerical tests unless specified otherwise.

The pseudo-frequency boundary data is obtained by taking the Laplace transform of the noisy data $u^{\delta}(x, t)$ as in (2.12). The Laplace transform is performed numerically using rectangular quadrature rule with a small time stepsize $\Delta t=5 \times 10^{-4}$. No filter is introduced during the collection of boundary data or the process of integration for the Laplace transform. The pseudo-frequency stepsize is chosen to be $h=0.05$. From our numerical tests we have observed an optimal pseudo-frequency interval $[4,6]$, namely $\underline{s}=4$ and $\bar{s}=6$.

We then take the pseudo-frequency boundary data to solve the inverse problem with our newly proposed inversion algorithm. Subproblems (2.7), (3.8)-(3.10), and (4.8)-(4.9) involved in our algorithm, which are all time independent, are solved using the second order central difference scheme with a mesh size $\Delta x=1 / 32$, since the coefficients $\mu_{n}$ during the iteration are smooth due to the smoothing step (5.1). As it is well known that the considered inverse problem is highly nonlinear and ill-posed, it cannot be solved using a mesh which is too fine due to the 
presence of noise in the observed data. From our numerical experiments, we observe that mesh size finer than 1/32 makes the inverse problem more unstable and ill-conditioned, leading to the approximate solutions with less accuracy, while the choice of the mesh size for $1 / 32$ seems to give reasonable reconstructions.

Next we present the reconstruction results for three test problems. In all the tests the inclusions are small rectangles with side length 0.1 and width 0.125 sitting inside the sampling region $\Omega$.

Example 1. We have a single inclusion sitting in the center of the domain $\Omega$, with its coefficient being $\mu(x)=0.5$, see Figure 1 (left). Figure 1 (right) shows the numerically reconstructed image, with a relative $L^{2}$-norm error of $7.35 \%$. As we can see, both the location of the inclusion and the magnitude of $\mu$ are recovered quite well, in view of the strong nonlinearity and severe ill-posedness of the inverse problem.
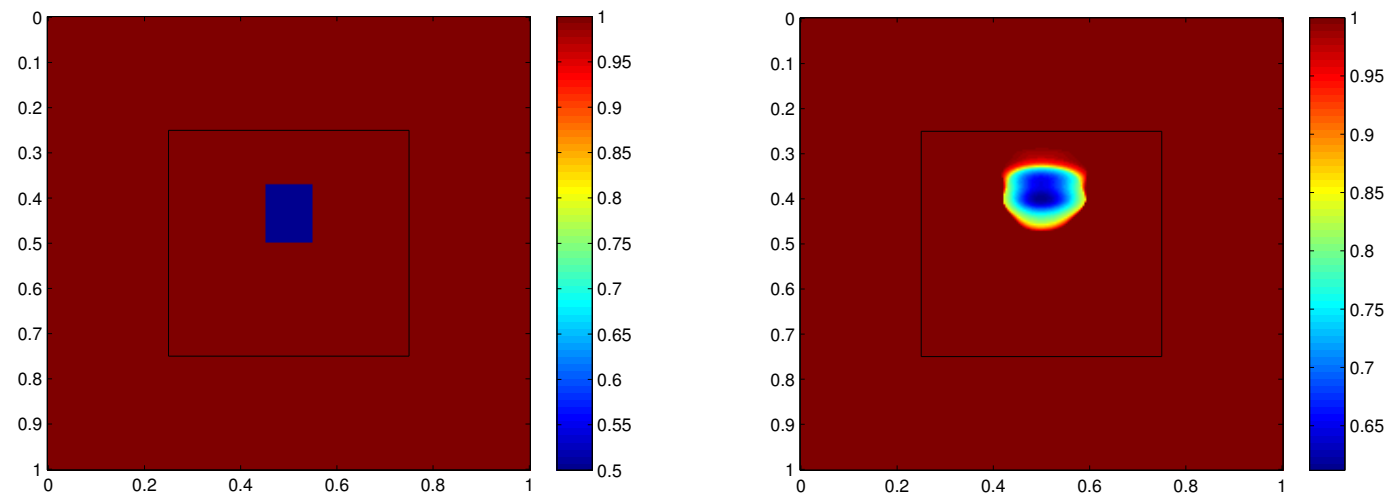

Figure 1: Exact image (left) and reconstructed image (right) for Example 1.

Example 2. We have the same inclusion as in Example 1, but it is placed very close to the right boundary of the sampling domain; see Figure 2 (left). The reconstruction image is shown in Figure 2 (right), with a relative $L^{2}$-norm error of $4.30 \%$. The same as in Example 1, both the location of the inclusion and the magnitude of $\mu$ are recovered quite well.
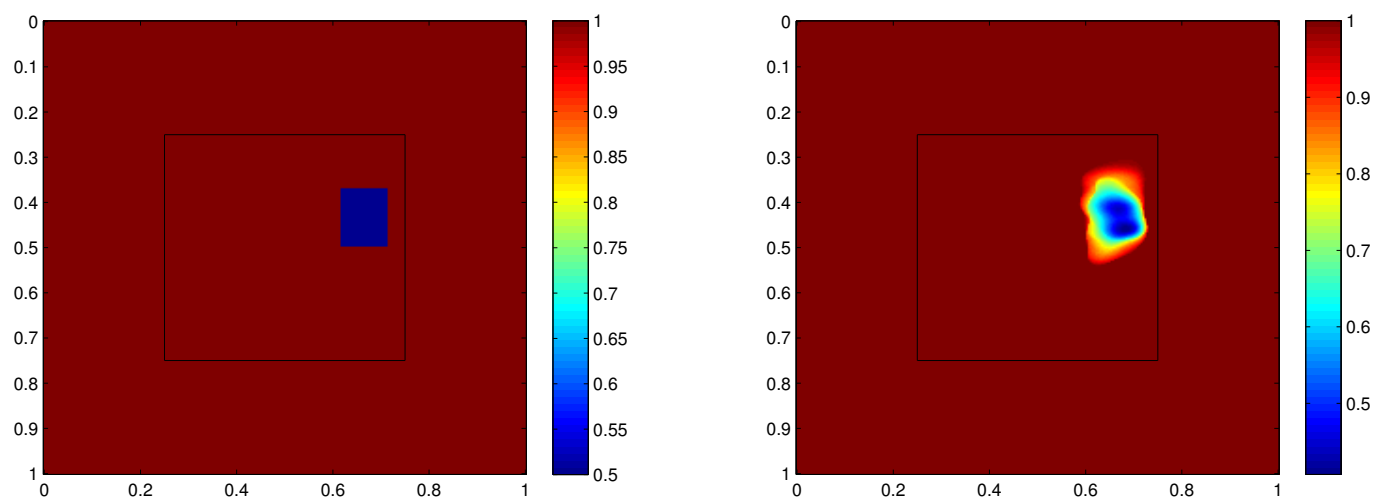

Figure 2: Exact image (left) and reconstructed image (right) for Example 2. 
Next, we would like to use this example to illustrate and investigate the effects of the choice of various parameters involved on numerical reconstructions.

Effect of parameter $\bar{s} . \bar{s}$ is referred to as a regularization parameter in section 3 . Theoretically speaking, the asymptotic formula (3.2) tells that, as the pseudo-frequency $s$ grows larger and larger, the term $(-1)^{k} \frac{p(x)}{s^{k+1}}$ shall better approximate the term $\partial_{s}^{k} v$ for $k=0,1$. Hence the approximate model (4.8)-(4.9), which is derived based on this approximation and gives the first initial guess for $V_{1}$ required in our inversion algorithm, should be more and more accurate as $s$ goes larger. In general, we should expect better reconstruction with higher pseudo-frequency. However, with a rapid decreasing of the kernel $e^{-s t}$ in the Laplace transform, a quadrature rule with very high order (thus very expensive and often more unstable numerically, especially against the noise) is needed to do the Laplace transform numerically when the cut-off vale $\bar{s}$ becomes very large. Therefore we should take $\bar{s}$ appropriately and not take it to be too large. In this sense, $\bar{s}$ can be viewed as a regularization parameter in our inversion algorithm.

In order to sort out the best choice of $\bar{s}$, numerical experiments have been conducted to observe the effects of different choices of $\bar{s}$ on the numerical reconstructions, with $\bar{s}$ varying among $\bar{s}=10,9,8, \ldots, 3,2,1$; see Table 1 and Figure 3 . We can see that the numerical construction becomes less accurate when $\bar{s}$ increases over 6 . It is the best when $\bar{s}$ is from 4 to 6 . When $\bar{s}$ is down to 3 , the approximation (4.8)-(4.9) is inaccurate for $V_{1}$, leading to a poor reconstruction by our proposed method. For $\bar{s}=1,2$, the iterations do not even converge due to the fact that the approximation (4.8)-(4.9) is far from being accurate.

\begin{tabular}{|c|c|c|}
\hline $\bar{s}$ & relative $L^{2}$-norm error & minimum value of $\mu$ in the recovered inclusion \\
\hline 10 & $6.91 \%$ & 0.7308 \\
\hline 9 & $7.28 \%$ & 0.7418 \\
\hline 8 & $7.17 \%$ & 0.7266 \\
\hline 7 & $6.93 \%$ & 0.6988 \\
\hline 6 & $6.24 \%$ & 0.6399 \\
\hline 5 & $4.30 \%$ & 0.4069 \\
\hline 4 & $5.68 \%$ & 0.5388 \\
\hline 3 & $10.30 \%$ & 0.3428 \\
\hline 1,2 & diverge & diverge \\
\hline
\end{tabular}

Table 1: Relative $L^{2}$-norm error and recovered $\mu$ for Example 2, with $\bar{s}=10,9, \ldots, 1$.

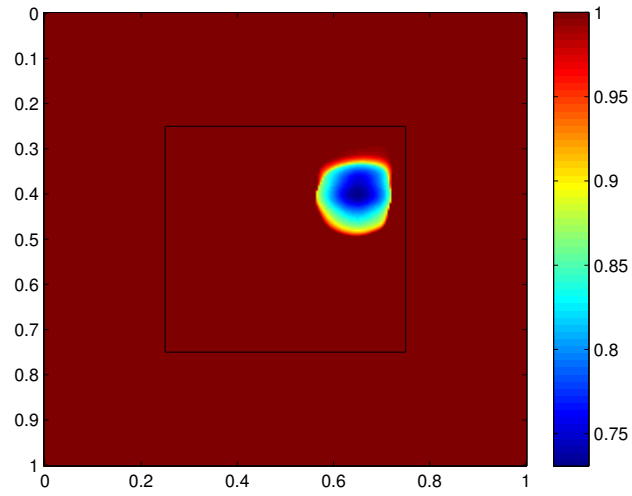

(a)

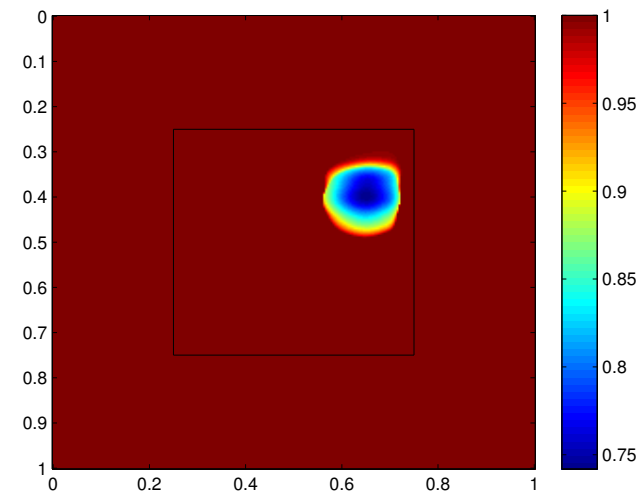

(b) 


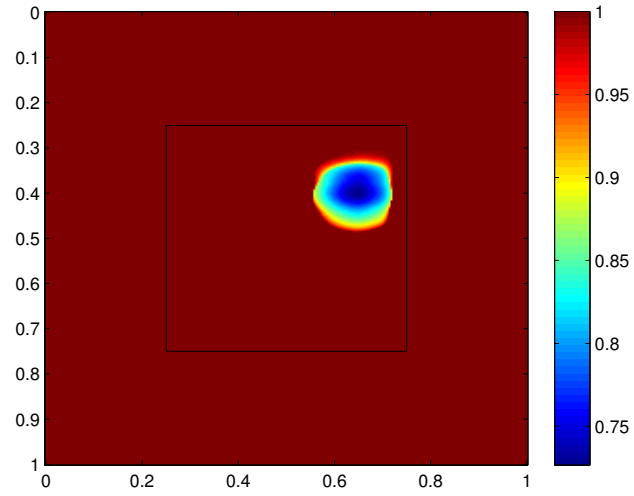

(c)

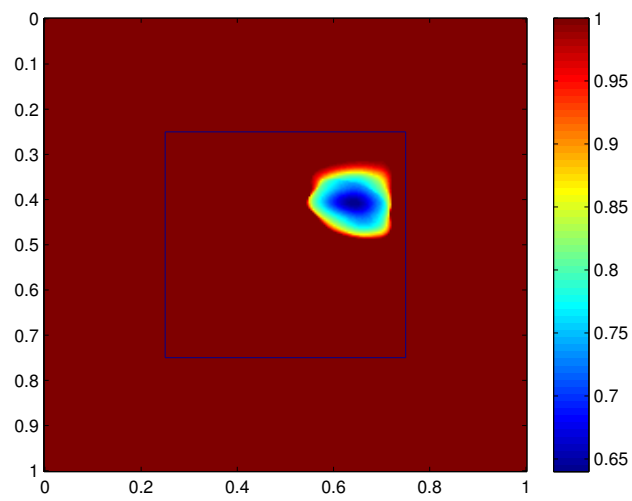

(e)

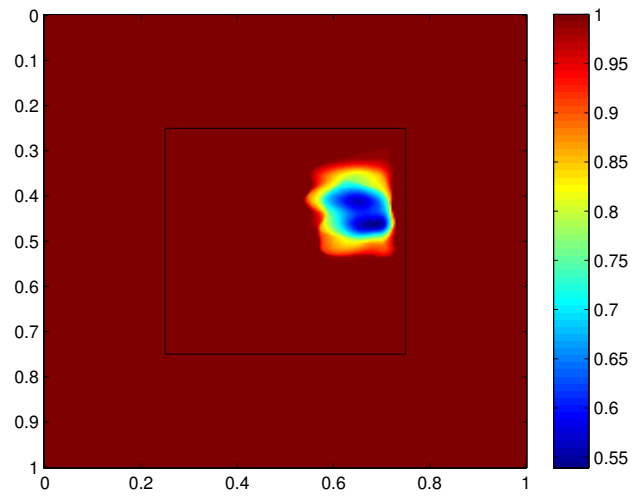

(g)

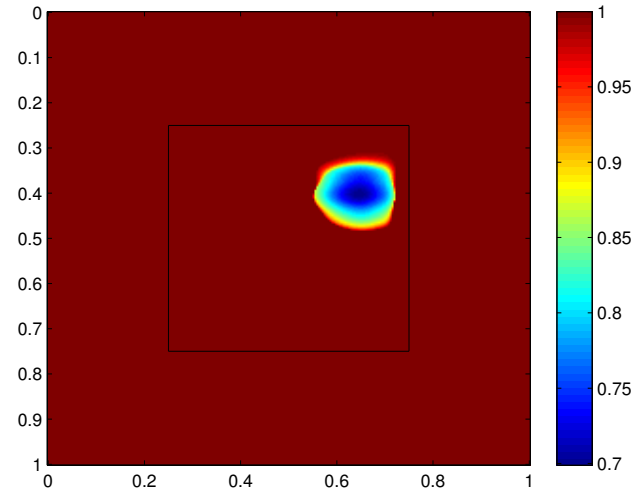

(d)

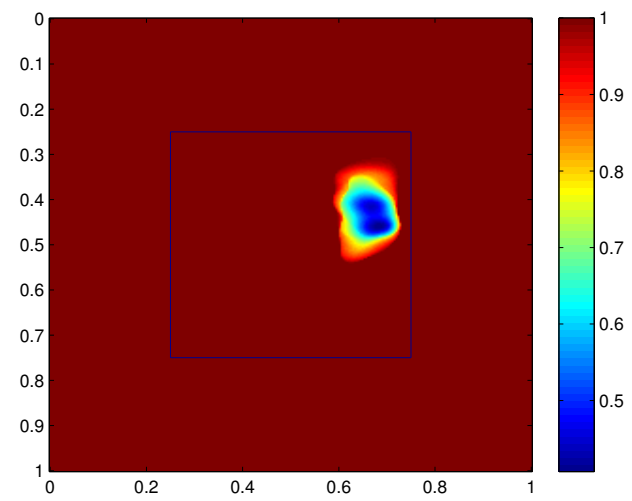

(f)

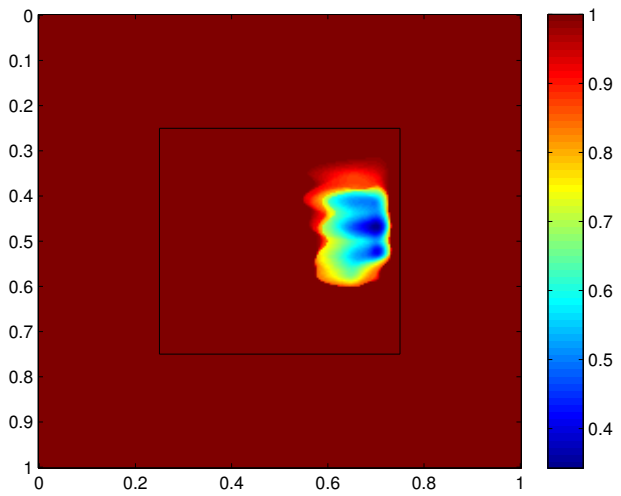

(h)

Figure 3: Reconstructed images for Example 2, with $\bar{s}=10,9, \ldots, 3$, from (a) to (h) respectively.

Effect of the choice of ad-hoc boundary data. The ad-hoc boundary data (3.10) and (4.9) are frequently used in the inverse wave problem $[1,2,3]$. We have performed numerical simulations to compare the reconstructions by our proposed method with two different sets of observed boundary data:

(A1) full boundary data, namely the observation data on the entire boundary $\partial \Omega$; 
(A2) backscattering data on $\Gamma$ combined with the ad-hoc boundary data (3.10), (4.9) on $\partial \Omega \backslash \Gamma$.

The reconstructions are shown in Table 2 and Figure 4, from which we can see that the reconstructions with two set of observed boundary data do not present much difference. This indicates that the ad-hoc boundary data (3.10), (4.9) do not affect the numerical reconstruction results much, while the backscattering data plays a major role.

\begin{tabular}{|c|c|c|}
\hline boundary data & relative $L^{2}$-norm error & minimum value of $\mu$ in the recovered inclusion \\
\hline (A1) & $4.38 \%$ & 0.4090 \\
\hline (A2) & $4.30 \%$ & 0.4069 \\
\hline
\end{tabular}

Table 2: Relative $L^{2}$-norm error and recovered $\mu$ for Example 2, with boundary data (A1) and (A2).
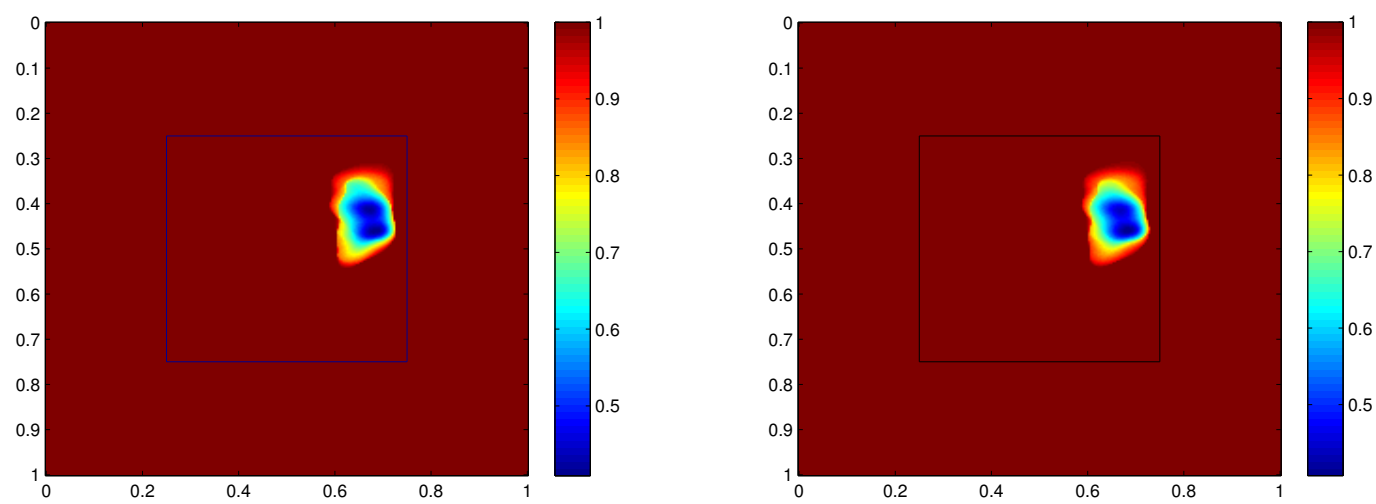

Figure 4: Reconstructed images for Example 2 with boundary data (A1) (left) and (A2) (right).

Effect of the number of measurement points. To see the effect of the number of measurement points on the numerical reconstruction, we take the number of the measurement points on $\Gamma$ to be $2^{n}+1$, with $n=2,3, . ., 5$. We observe from Table 3 and Figure 5 that the reconstructed images are of less accuracy if fewer measurement points are used: the size, position and contrast of the recovered inclusion are all less accurate. As the number of points grows, the resolutions of the reconstructions increase. Moreover, the size and the location of the inclusion as well as the contrast becomes more accurate as more number of points are taken.

\begin{tabular}{|c|c|c|}
\hline number of measurement points & relative $L^{2}$-norm error & minimum value of $\mu$ in the recovered inclusion \\
\hline 5 & $8.09 \%$ & 0.7817 \\
\hline 9 & $7.49 \%$ & 0.6988 \\
\hline 17 & $4.30 \%$ & 0.4069 \\
\hline 33 & $4.61 \%$ & 0.5003 \\
\hline
\end{tabular}

Table 3: Relative $L^{2}$-norm error and recovered $\mu$ for Example 2, with $2^{n}+1$ measurement points for $n=2,3, . ., 5$. 


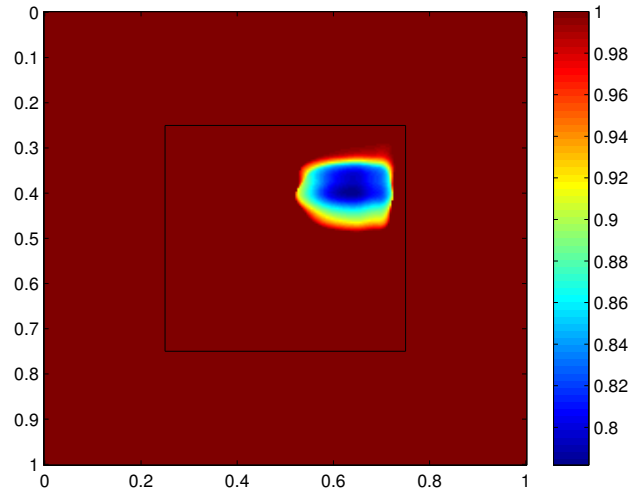

(a)

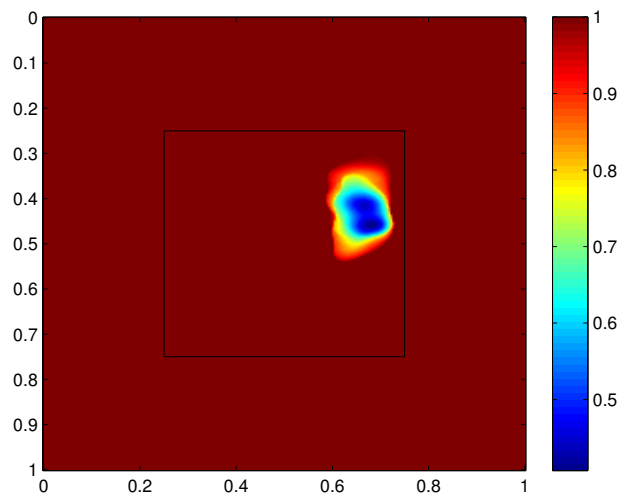

(c)

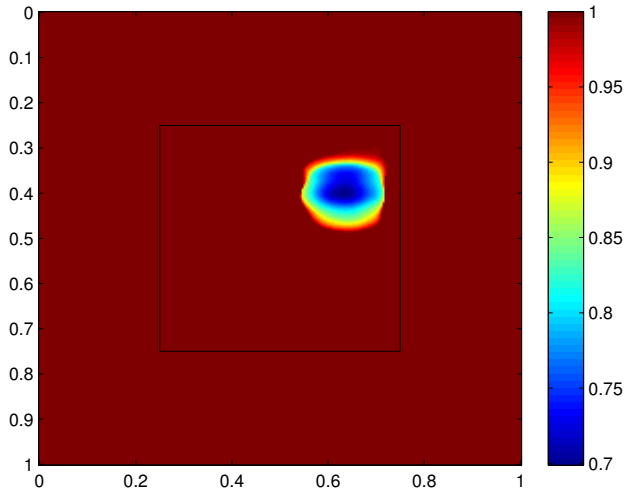

(b)

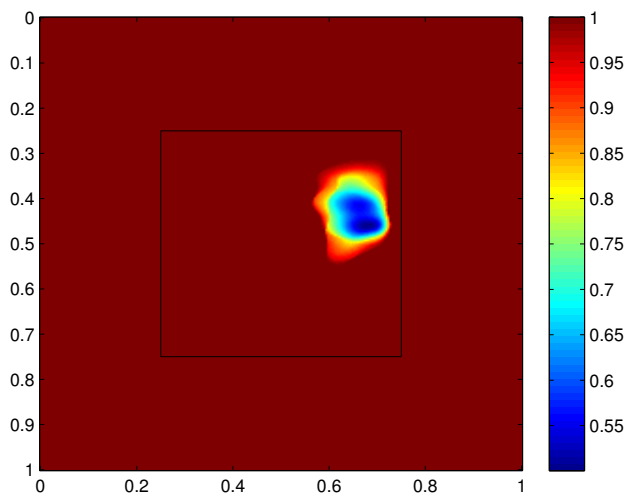

(d)

Figure 5: Reconstructed images for Example 2 with $2^{n}+1$ measurement points, $n=2,3, . ., 5$, from (a) to (d) respectively.

Effect of the choice of boundary conditions for the forward problem. For the inversion process, we need to first solve the forward problem (1.1) on the entire space $\mathbb{R}^{d}$ to generate the observation data. To do so, we should truncate the infinite domain $\mathbb{R}^{d}$, approximated by a finite computational domain $G$, and introduce some boundary conditions on the artificial boundaries of $G$. Most numerical tests are based on the boundary conditions specified in (6.1). Next, we shall test and compare the effects of the choice of the following two different sets of boundary conditions for the equation (1.1):

(B1) absorbing boundary conditions on the top and bottom boundaries and Neumann conditions on the left and right boundaries of the computational domain $G$, as specified in (6.1);

(B2) absorbing boundary condition on the top boundary and Neumann conditions on the remaining boundaries of the computational domain $G$.

The reconstructions are shown in Table 4 and Figure 6, from which we can see there are no essential differences between the reconstructions from these two different sets of boundary conditions. 


\begin{tabular}{|c|c|c|}
\hline boundary conditions & relative $L^{2}$-norm error & minimum value of $\mu$ in the recovered inclusion \\
\hline (B1) & $4.30 \%$ & 0.4069 \\
\hline (B2) & $4.35 \%$ & 0.4693 \\
\hline
\end{tabular}

Table 4: Relative $L^{2}$-norm error and recovered $\mu$ for Example 2, with boundary conditions (B1) and (B2).
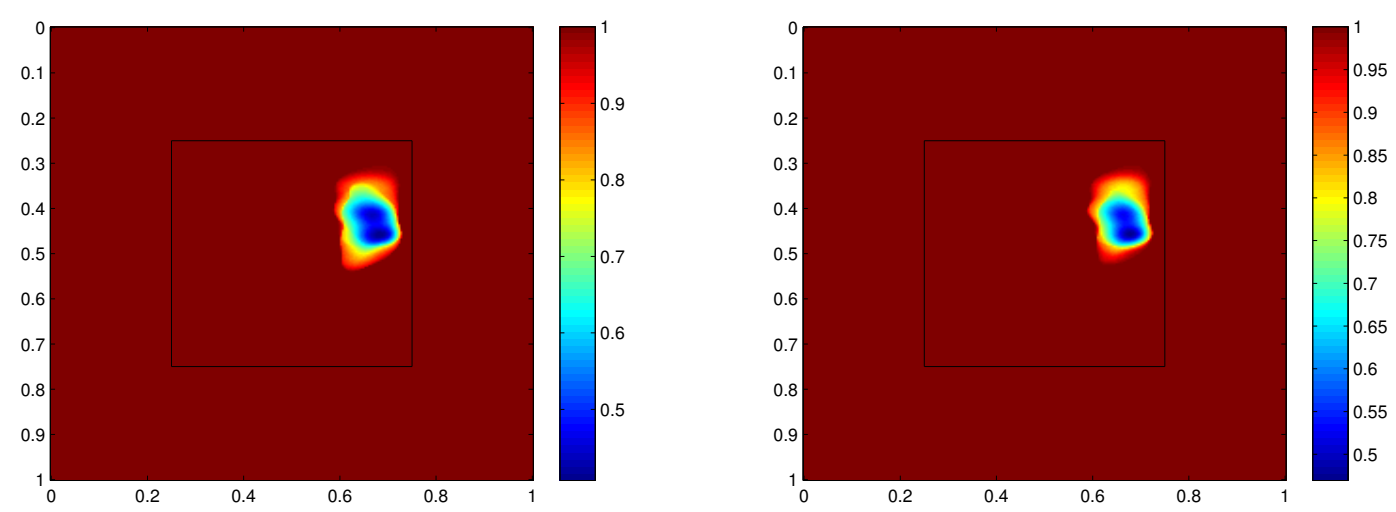

Figure 6: Reconstructed images for Example 2, with two different boundary conditions (B1) (left) and (B2) (right).

Effect of the value of coefficient $\mu$ in the inclusion. In this test we shall see the effect of the value of coefficient $\mu$ in the inclusion on the quality of numerical reconstructions. We take the same configuration of the inclusion as in Example 2, but with different coefficient $\mu$ inside the inclusion, and the numerical results are shown in Table 5 and Figure 7 . These results indicate that the quality of the reconstruction for both the location and the values of $\mu$ in the inclusions does not deteriorate much if the values of $\mu$ in the inclusion differ from the one in the background only by a few times in magnitude.

\begin{tabular}{|c|c|c|}
\hline exact $\mu$ in the inclusion & relative $L^{2}$-norm error & minimum value of $\mu$ in the recovered inclusion \\
\hline $3 / 4$ & $3.63 \%$ & 0.7826 \\
\hline $1 / 2$ & $4.30 \%$ & 0.4069 \\
\hline $1 / 3$ & $8.04 \%$ & 0.3674 \\
\hline $1 / 4$ & $10.06 \%$ & 0.2883 \\
\hline
\end{tabular}

Table 5: Relative $L^{2}$-norm error and recovered $\mu$, with exact $\mu=\frac{3}{4}, \frac{1}{2}, \frac{1}{3}, \frac{1}{4}$ inside the inclusion. 

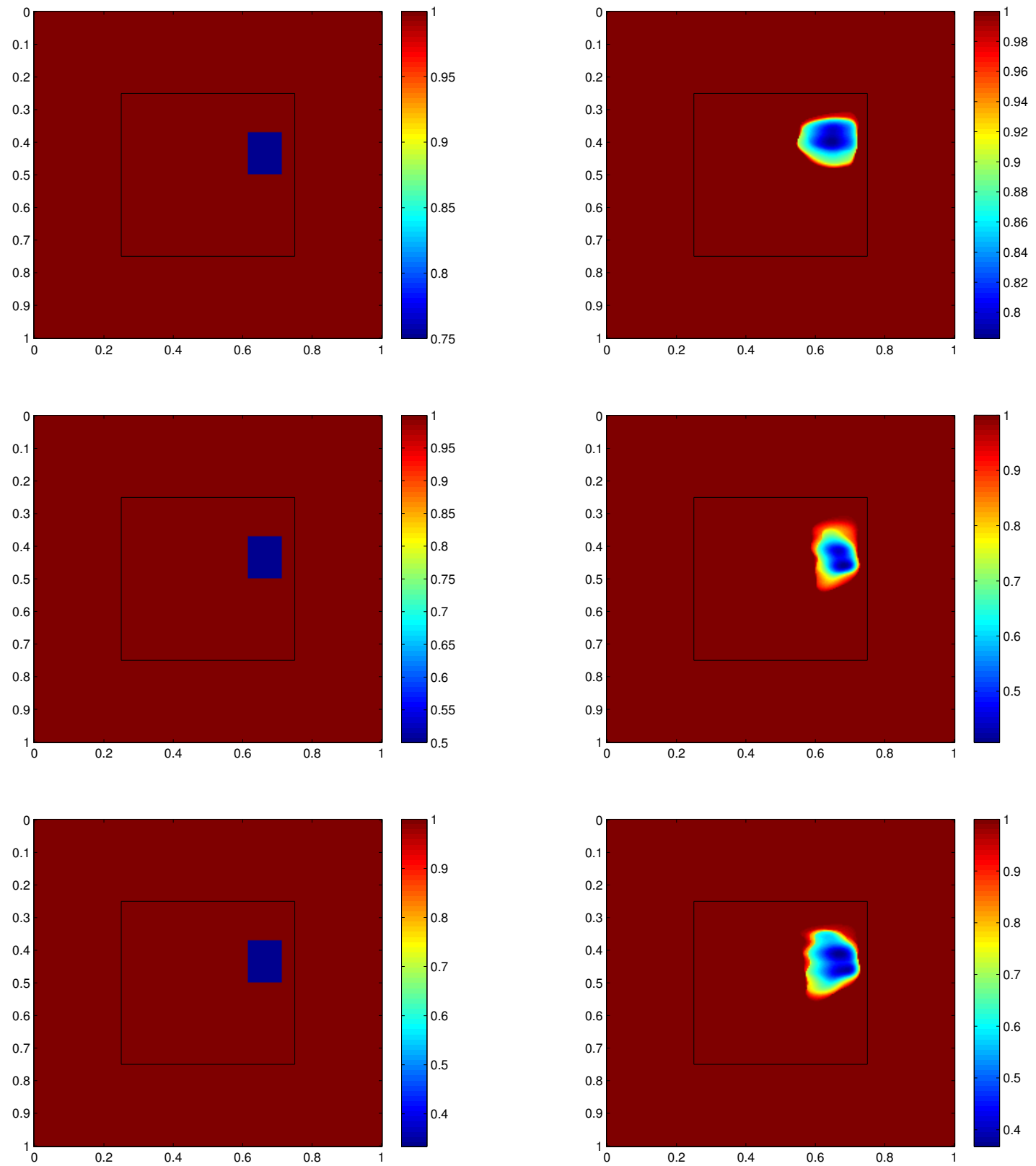

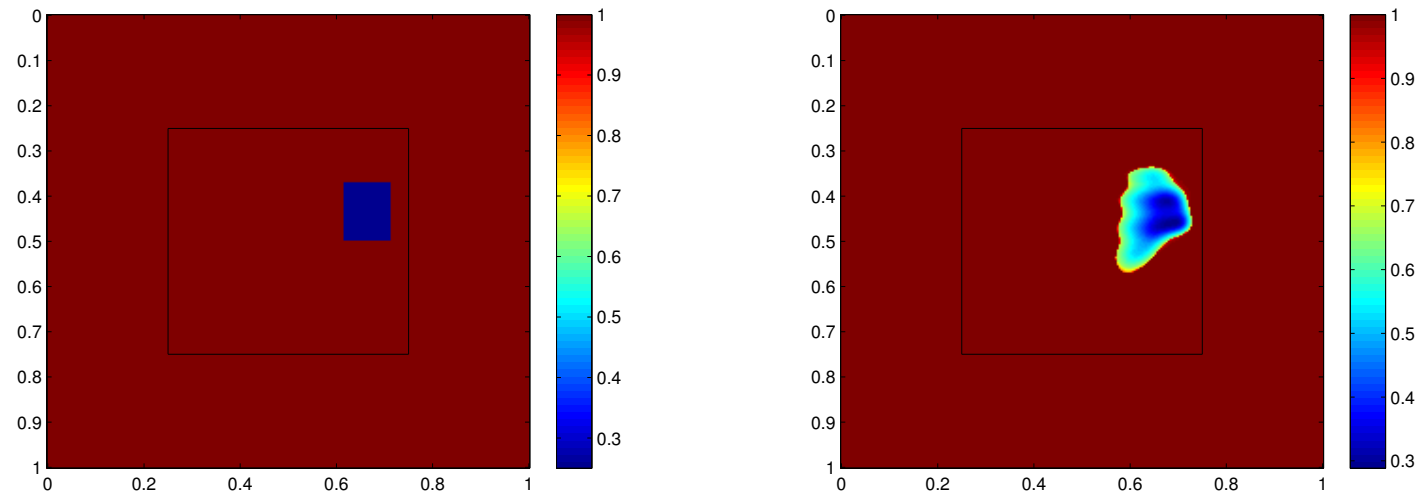

Figure 7: Exact images with $\mu=\frac{3}{4}, \frac{1}{2}, \frac{1}{3}, \frac{1}{4}$ inside the inclusion (left) and the respectively reconstructed images (right).

Effect of the medium noise on numerical reconstructions. Finally, we come to test the effect of the medium noise on numerical reconstructions. We introduce some multiplicative medium noise to the coefficient $\mu$ in Example 2 as follows:

$$
\mu^{\delta}(x)=\mu(x)(1+\varepsilon \zeta(x)) \quad \forall x \in G,
$$

where $\varepsilon=5 \%$ is the noise level, and $\zeta(x)$ is a random variable uniformly distributed in $[-1,1]$; see Figure 8 (left). The forward data is then calculated by solving (6.1) with coefficient $\mu^{\delta}$. Then multiplicative noise is added to the data $u(x, t)$ along $\Gamma$ in the time domain as in (6.2) and is taken to solve the inverse problem with our newly proposed algorithm. The reconstruction of the medium coefficient is given in Figure 8 (right) and Table 6. We can see that, although the reconstruction of the medium is contaminated by $5 \%$ medium noise and is worse than the original reconstruction, the overall profile still stands out quite clearly, and both the location and the contrast of the inclusion is still reconstructed with certain accuracy.

\begin{tabular}{|c|c|c|}
\hline & relative $L^{2}$-norm error & minimum value of $\mu$ in the recovered inclusion \\
\hline Exact medium $\mu$ & $4.30 \%$ & 0.4069 \\
\hline Noisy medium $\mu^{\delta}$ & $8.82 \%$ & 0.3806 \\
\hline
\end{tabular}

Table 6: Relative $L^{2}$-norm error and recovered coefficient for Example 2, with exact media $\mu$ and noisy $\mu^{\delta}$ respectively. 

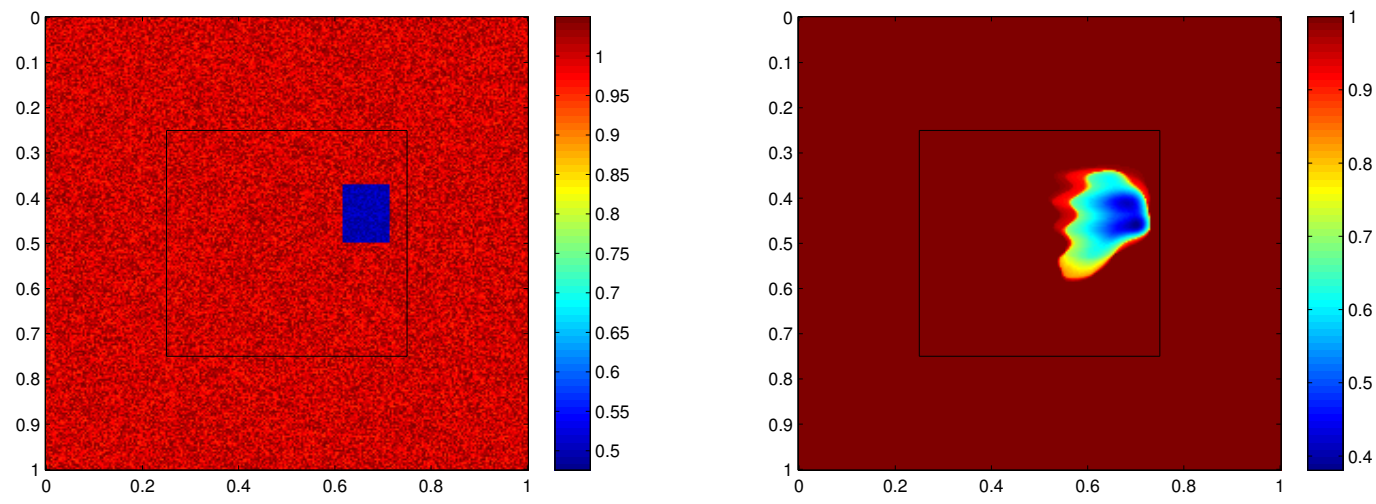

Figure 8: Image of noisy medium $\mu^{\delta}$ (left) and reconstructed image (right) for Example 2.

Example 3. This example considers two inclusions, which are placed separately on a row in the sampling domain $\Omega$, with their coefficient values being $\mu=0.33$ and $\mu=0.5$ respectively. see Figure 9 (left). The reconstructed inclusions are shown in Figure 9 (right), with a relative $L^{2}$ norm error of $11.04 \%$. We can see that both the locations and abnormalities of two inclusions are recovered with an acceptable accuracy, considering the high nonlinearity and severe ill-posedness of the inverse problem and a $5 \%$ multiplicative noise in the data.
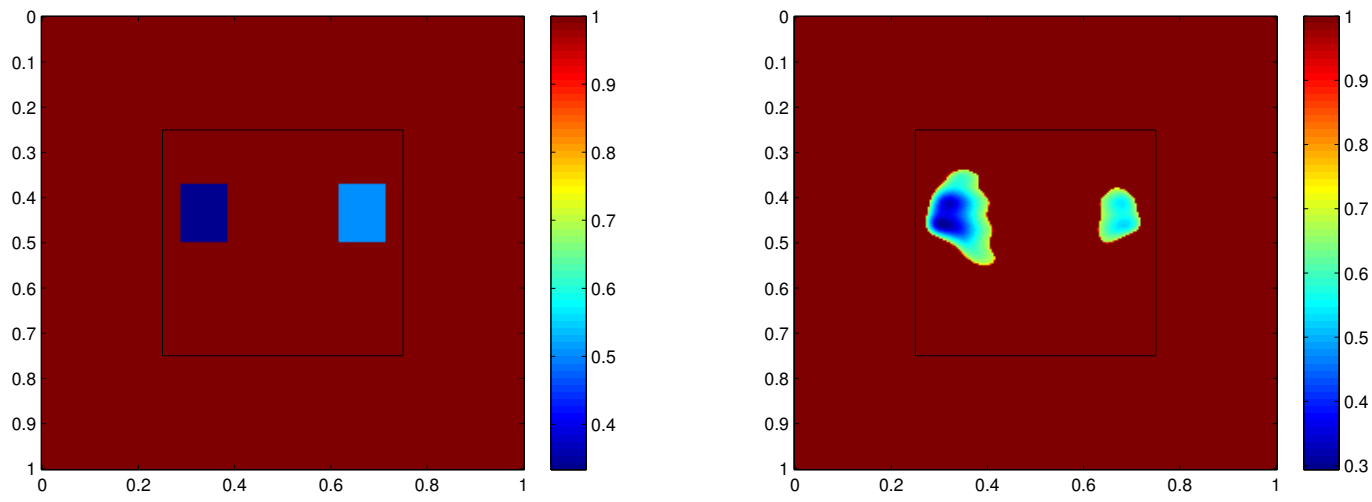

Figure 9: Exact image (left) and reconstructed image (right) for Example 3.

Convergence rate and iterative counts. As our last test, we shall see a bit more on the convergence rate and iteration counts of our proposed inversion algorithm in the aforementioned 3 examples. Table 7 shows the iteration counts of our proposed algorithm for the 3 aforementioned examples. From these data, we can see that the average number of outer loops to achieve convergence is about 10 to 12 . Convergence speed is also investigated by considering the relative residual of the $n$-th iterate $\mu_{n}$ when compared with the measured data (with $5 \%$ noise); see Figure 10. Although the considered inverse problem is highly nonlinear and severely ill-posed, we can still see the sublinear convergence in all the examples. 


\begin{tabular}{|c|c|c|}
\hline example & outer loops & average inner loops per outer loop \\
\hline 1 & 12 & 4.5 \\
\hline 2 & 10 & 7.9 \\
\hline 3 & 10 & 9 \\
\hline
\end{tabular}

Table 7: Iteration counts of the new inversion algorithm for the 3 examples
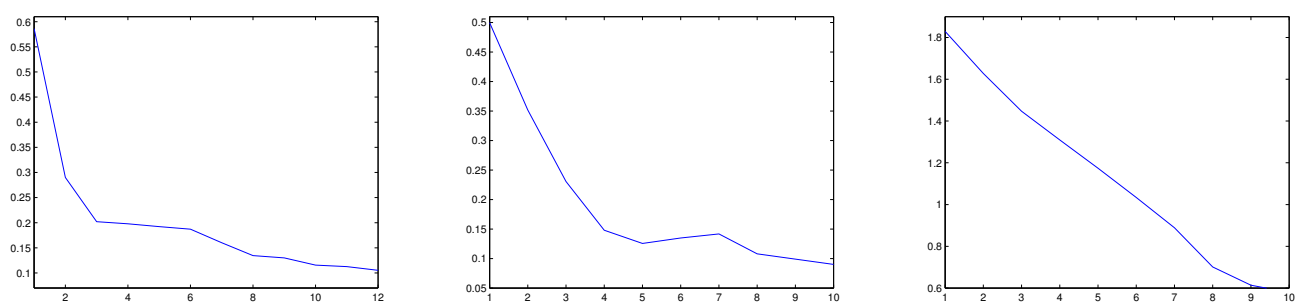

Figure 10: Relative residual with respect to number of outer loops for Examples 1 (left), 2 (middle) and 3 (right).

\section{Acknowledgements}

The authors would like to thank two referees for their many insightful and constructive comments and suggestions, which have helped us improve the organisation and quality of the paper essentially. The authors are particularly grateful to L. Beilina and M.V. Klibanov for their great help during the algorithmic development of this work and for their numerous instructive and insightful suggestions and advices in understanding their approximate globally convergent algorithm. The work of Jun Zou was substantially supported by Hong Kong RGC grants (Projects 405110 and 404611).

\section{References}

[1] L. Beilina and M.V. Klibanov, A globally convergent numerical method for a coefficient inverse problem, SIAM J. Sci. Comp., 31, (2008), pp. 478-509.

[2] L. Beilina and M.V. Klibanov, A new approximate mathematical model for global convergence for a coefficient inverse problem with backscattering data, J. Inv. Ill-Posed Probl., 20, (2012), pp. 513-565.

[3] L. Beilina and M.V. Klibanov, Approximate Global Convergence and Adaptivity for Coeffcient Inverse Problems, Springer, New York (2012).

[4] C. Bellis, M. Bonnet, B.B. Guzina. Apposition of the topological sensitivity and linear sampling approaches to inverse scattering, Wave Motion, 50, (2013), pp. 891-908.

[5] G. Berylkin, R. Burridge, Linearized inverse scattering problems in acoustic and elasticity, Wave Motion, 12, (1990), pp. 15-52. 
[6] H.D. Bui, A. Constantinescu, and H. Maigre, An exact inversion formula for determining a planar fault from boundary measurements, J. Inv. Ill-Posed Probl., 13(6), (2005), pp. 553-565.

[7] H.D. Bui, A. Constantinescu, and H. Maigre, Numerical identification of planar cracks in elastodynamics using the instantaneous reciprocity gap, Inverse Problems, 20, (2004), pp. 993-1001.

[8] Z. Chen and J. Zou, Finite element methods and their convergence for elliptic and parabolic interface problems, Numerische Mathematik, 79, (1998), 175-202.

[9] R.W. Clayton, R. H. Stolt, A Born-WKBJ inversion method for acoustic reflection data, Geophysics, 46(11), (1981), pp. 1559-1567

[10] H.W. Engl, M. Hanke and A. Neubauer, Regularization of Inverse Problems, Kluwer Academic Publishers, Boston, (2000).

[11] B. Engquist and A. Majda, Absorbing boundary conditions for the numerical simulation of waves, Math. Comp., 31, (1977), pp. 629-651.

[12] S. N. Fata, B.B. Guzina, A linear sampling method for near-field inverse problems in elastodynamics, Inverse Problem 20 (2004), pp. 713-736

[13] D.J. Griffiths, Introduction to Electrodynamics (3rd ed.), Prentice Hall, (1998).

[14] A.J. Robins, Reflection of plain acoustic waves from a layer of varying density, J. Acoust. Soc. Am., 87(4), (1990), pp. 1546-1552.

[15] A. Tarantola, Inversion of seismic reflection data in the acoustic approximation, Geophysics, 49(8), (1984), pp. 1259-1266. 